\title{
Delocalization and New Phase in Americium: Theory
}

\author{
Per Soderlind
}

April 23, 1999

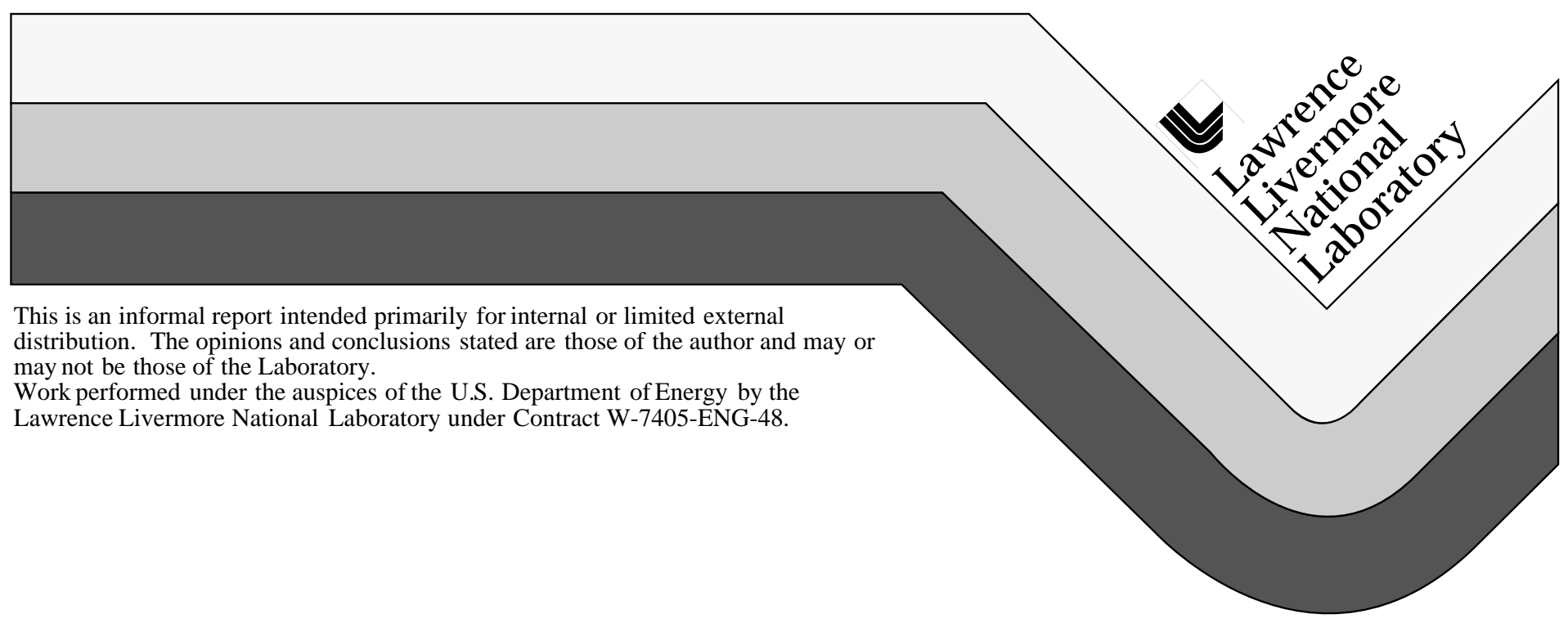




\section{DISCLAIMER}

This document was prepared as an account of work sponsored by an agency of the United States Government. Neither the United States Government nor the University of California nor any of their employees, makes any warranty, express or implied, or assumes any legal liability or responsibility for the accuracy, completeness, or usefulness of any information, apparatus, product, or process disclosed, or represents that its use would not infringe privately owned rights. Reference herein to any specific commercial product, process, or service by trade name, trademark, manufacturer, or otherwise, does not necessarily constitute or imply its endorsement, recommendation, or favoring by the United States Government or the University of California. The views and opinions of authors expressed herein do not necessarily state or reflect those of the United States Government or the University of California, and shall not be used for advertising or product endorsement purposes.

This report has been reproduced directly from the best available copy.

Available to DOE and DOE contractors from the Office of Scientific and Technical Information P.O. Box 62, Oak Ridge, TN 37831

Prices available from (423) 576-8401

Available to the public from the National Technical Information Service

U.S. Department of Commerce 5285 Port Royal Rd. Springfield, VA 22161 
Draft, not for distribution

\title{
Delocalization and new phase in americium: theory
}

\author{
Per Söderlind \\ Department of Physics, Lawrence Livermore National Laboratory, \\ Livermore, California 94550
}

(April 23, 1999)

\begin{abstract}
Density-functional electronic structure calculations have been used to investigate the high pressure behavior of Am. At about $80 \mathrm{kbar}(8 \mathrm{GPa}$ ) calculations reveal a monoclinic phase similar to the ground state structure of plutonium $(\alpha-\mathrm{Pu})$. The experimently suggested $\alpha$-U structure is found to be substantially higher in energy. The phase transition from fcc to the low symmetry structure is shown to originate from a drastic change in the nature of the electronic structure induced by the elevated pressure. A calculated volume collapse of about $25 \%$ is associated with the transition. For the low density phase, an orbital polarization correction to the local spin density (LSD) theory was applied. Gradient terms of the electron density were included in the calculation of the exchange/correlation energy and potential, according to the generalized gradient approximation (GGA). The results are consistent with a Mott transition; the $5 f$ electrons are delocalized and bonding on the high density side of the transition and chemically inert and non-bonding (localized) on the other. Theory compares rather well with recent experimental data which implies that electron correlation effects are reasonably modeled in our orbital polarization scheme.
\end{abstract}

71.10.+x., 71.25.Pi.,71.28.+d.

Typeset using REVTEX 


\section{INTRODUCTION}

Americium belongs to the series of actinide metals. These elements have received considerable interest because of their nuclear properties but also because of their fascinating ground state electronic properties. The latter is perhaps best illuminated by their crystal structure and atomic volume behavior. ${ }^{1}$ The crystal structures of the actinides are different from almost any other metal in the periodic table. Although the first two actinides, Ac and Th, have the fcc crystal structure, the crystal structures of the next four; Pa, $\mathrm{U}, \mathrm{Np}$, and $\mathrm{Pu}$, show an increasing complexity with plutonium attaining a monoclinic (16 atoms/cell) structure. Continuing in the actinide series a most peculiar observation is made, the crystal structure of Am (next to $\mathrm{Pu}$ ) has a closed packed crystal structure (dhcp) similar to the structure of the rare-earth metals and not at all similar to the open and low symmetry structures exhibited by the preceding actinides. The metals beyond Am also follow the example of Am with high symmetry and close packed structures. Hence, the trend in the crystal structures is completely broken down between Pu and Am. As regards the atomic volumes, the behavior of the light actinides, up to $\mathrm{P} u$, is similar to the non-magnetic $d$ transition metals, the atomic volume is decreasing in a parabolic manner $^{2}$ as we proceed through Th, Pa, U, Np, and Pu. Actually, the volume of $\mathrm{Pu}$ is almost identical with to that of Np. Again, we find a complete break-down of this trend when continuing through Am, Bk, and Cf. The atomic volume of Am is about $40 \%$ larger than that of $\mathrm{Pu}$ and the following metals have also a much lower density than $\mathrm{U}, \mathrm{Np}$, and Pu. Thus, the trend of two important ground state properties, the crystal structure and the atomic volume, display a most obvious interruption between $\mathrm{Pu}$ and $\mathrm{Am}$. Am and the metals beyond form a second rare-earth series. Another key distinction between the itinerant and localized regimes could be attributed to the possible formation of magnetic moments on the $f$-electron sites. Generally the localized phase of an $f$-electron metal show magnetic ordering at low temperatures according to Russel-Saunders coupling for a free ion. However for Am, the $f^{6}(J=0)$ ion configuration cancel the magnetic moment so that the localized phase could not be distinguished from the itinerant phase by considering their respective magnetic properties.

The actinide metals are the first elements to populate the $5 f$ orbitals in the periodic table. None of the electrons in Ac occupy a $5 f$ state but Th has a non-negligible amount of $5 f$ states, but the next element filled.,3 Proceeding through the actinide series the $5 f$ occupation is increasing with about one electron per element and $\mathrm{Pu}$ has a total of about five and Am about six $5 f$ states filled. ${ }^{1}$ The experimental observations outlined in the previous paragraph could best be explained by a dramatic change in the electronic structure between $\mathrm{Pu}$ and Am. Specifically, it is believed that the $5 f$ electrons of the lighter actinides, Th-Pu, have metallic or itinerant character greatly influencing the bonding characteristics, whereas for the heavier actinides, Am and on, the $5 f$ electrons are localized and of minor importance for the chemical bond between the atoms in the solid. This makes sense because, first, the crystal structures of Am, Bk, and Cf are very similar to the rare-earth crystal structures, which in turn has been shown to originate from the bonding characteristics of their $d$ electrons. ${ }^{4}$ Secondly, the parabolic decrease of the atomic volume of the actinides up to $\mathrm{Pu}$ could be understood from consecutive filling of bonding (itinerant) $5 f$ states gradually increasing the interatomic bonds through 
Th- $\mathrm{Pu}$, whereas the jump in volume to Am could be explained by the removal of some of the contribution to the chemical bonding between the atoms. Hence, there is a transition between $\mathrm{Pu}$ and $\mathrm{Am}$ that originates from a localization of the $5 f$ electrons, ${ }^{5}$ a so-called Mott transition. In fact, it has been argued ${ }^{6}$ that such transitions take place, partially or completely, already in the phase diagram of $\mathrm{Pu}$. At higher temperatures and expanded volumes a fcc phase $(\delta)$ of $\mathrm{Pu}$ shows similarities to both $\alpha$-Pu and Am and this may signal a Mott transition within Pu's phase diagram initiated by external parameters (pressure and temperature). This is to some extent in line with the picture of a Mott transition in Am (delocalization of the $5 f$ electrons), induced by external pressure. Experimentally this has also been suggested in connection to the discovery of low symmetry phase in Am at elevated pressure.

The experimental situation has been somewhat confusing for Am arising from discrepancies in x-ray experiments. The equilibrium, dhcp, structure has not been a subject of controversy, but the second phase, fcc, has been observed at different pressures. Akella et al. ${ }^{7}$ found a $50 \%$ mixture of dhep and fcc at $52 \mathrm{kbar}(5.2 \mathrm{GPa})$ and only fec at $65 \mathrm{kbar}$. Benedict et al. ${ }^{8}$, on the other hand, observed the dhcp up to 65 kbar. Later, Benedict and Dabos ${ }^{9}$ observed dhep up to $90 \mathrm{kbar}$ and fcc at $95 \mathrm{kbar}$. Benedict also reported the Am IV phase as an orthorhombic structure $\left(\alpha\right.$-U) at about 150 kbar. ${ }^{10}$ The most recent study, however, showed that at 63 kbar there was only an fcc phase in the Am sample. ${ }^{11}$ In this latter publication various results for the bulk modulus and its pressure derivative was quoted and an average of $\mathrm{B}_{0}=294 \mathrm{kbar}$ and $\mathrm{B}_{0}^{\prime}=3.0$ was calculated. These are the experimental results we have quoted in Table I. The third phase of Am (Am III) has proven to be very difficult to characterize and several structures have been proposed for this phase. Different monoclinic structures, trigonal distorted fcc, and orthorhombic structures have all been suggested for Am III. ${ }^{7-9,11}$ Heathman's experiments ${ }^{11}$ are the most recent and his study showed that $80 \%$ Am III was present at 91 kbar $(9.1 \mathrm{GPa})$ and only Am III at 100 kbar. At 110 kbar Am III was best fitted to the $\alpha$ ' structure (orthorhombic; $\alpha$-U) but was disregarded because that fit would result in a too small volume. Instead Heathman ${ }^{11}$ proposed the $\alpha$ " structure that has also been suggested for cerium. This is a monoclinic structure with two atoms per cell. The last phase, Am IV, was present $(10 \%)$ at $130 \mathrm{kbar}(13 \mathrm{GPa})$ and exclusively present at $175 \mathrm{kbar}$ in the study by Heathman and he concluded that this phase was the $\alpha$-U orthorhombic structure. Benedict came to the same conclusion ${ }^{10}$ and in his measurement the Am III $\rightarrow$ Am IV took place at $150 \mathrm{kbar}(15 \mathrm{GPa})$. Releasing the pressure, Am IV ( $\alpha$-U) and Am III (fcc) were present in equal amounts at $59 \mathrm{kbar}$ in the experiment by Heathman.

The motivation for the present study is mainly two-fold. First, we believe that the new phase in Am proposed experimentally ${ }^{11,10}(\alpha-\mathrm{U})$ is questionable. Recently ${ }^{12}$ the complex structures of the actinides were analyzed in terms of a simple model involving Pettifor's structural energy difference theorem. ${ }^{13}$ These model calculations suggested that a likely candidate for the new phase in Am would be the monoclinic structure of $\alpha$ $\mathrm{Pu}$ whereas the orthorhombic $\alpha$-U structure was less likely. In the present study we calculate the total energy for several crystal structures, including those of $\alpha$ - $\mathrm{U}$ and $\alpha$ $\mathrm{Pu}$, in order to investigate this aspect further. Secondly, previous attempts describing the Mott transition in Am from first-principles theory ${ }^{14,15}$ have been less satisfactory in reproducing the details of the experimental data. In the present study we use improved 
theory with better approximations for the exchange/correlation energy and potential and we also introduce an orbital polarization (OP) correction to the exchange/correlation functional. ${ }^{16,17}$ This scheme (GGA+OP) was applied for the Mott transition in $\operatorname{Pr}^{18}$ and compared favorably with the self-interaction corrected theory (LSD-SIC) and experiments. Generally, density functional calculations with a local spin density approximation are not able to accurately account for electron correlations that lead to localization effects of the electronic structure. This is a challenging problem and many attempts have been made to correct for this.

The localized (low density) phase of Am is here treated in the fcc crystal structure because this structure has been determined experimentally for moderate pressures of $\mathrm{Am}^{10}$ and the body of experimental data is consistent for this structure. The transition from dhcp to fcc has been shown to be related to the $d$ band occupation in Am and involves only a few meV and we do not consider this transition here. In the OP scheme the $5 f$ localization is associated with the onset of a nearly saturated magnetic spin and orbital moment. The spin polarization energy is included in the LSD and does not constitute a serious problem. The orbital polarization, however, is an effect that is present in open-shell Hartee-Fock theory and not in the LSD. Here this effect is included through an energy shift of the $5 f\left\{l, m_{l}, \sigma\right\}$ orbital equal to $-L_{\sigma} m_{l} E_{\sigma}^{3}$ where $L_{\sigma}$ is the orbital moment for spin channel $\sigma$ and $E_{\sigma}^{3}$ is the Racha parameter. When calculating the total energy an amount of $-\frac{1}{2} E_{\sigma}^{3} L_{\sigma}^{2}$ is added to correct for double counting. The fcc phase of Am was treated in this way, whereas the calculations of the other structures of Am were paramagnetic with no spin or orbital moments.

The following sections deals with computational details, results, and a discussion section.

\section{CALCULATION DETAILS}

The total energy for fcc, bcc, bcm $\left(\alpha^{\prime \prime}\right), \alpha$-U $\left(\alpha^{\prime}\right), \alpha$-Np, and $\alpha$-Pu structures ${ }^{19}$ of americium was calculated as a function of volume. The $\alpha^{\prime \prime}$ structure is a body centered monoclinic structure for which we chose $c / a=1.53$ and $b / a=1.03$ with the angle $\beta$ between the $a$ and $c$ axis equal to $92^{\circ}$. This is the same structure as was proposed by Olsen el al. ${ }^{20}$ in the phase diagram of cerium. For this we used the full potential version of the linear muffin-tin orbital method (FP-LMTO). ${ }^{21}$ This electronic structure method is an implementation of density functional theory as applied for a bulk material. It is a first-principle method, no experimental numbers are used in the calculations except for the nuclear charge which is 95 for Am. The approximations in this approach are limited to the approximation of the exchange/correlation energy functional, cut offs in the expansion of basis functions, k-point sampling in integrations over the Brillouin zone, and the BornOppenheimer approximation. For the exchange/correlation approximation we used the generalized gradient approximation (GGA) which has proven to be better for $f$-electron metals than the more commonly used local density approximations. In all calculations we used two energy tails associated with each basis orbital and for $6 s, 6 p$, and the valence states $(7 s, 7 p, 6 d$, and $5 f)$ these pairs were different. With this "double basis" approach we used a total of six energy tail parameters and a total of 12 basis functions per atom. Spherical harmonic expansions were carried out through $l_{\max }=6$ for the bases, potential 
and charge density. The sampling of the Brillouin-zone was done using the special $\mathrm{k}$ point method ${ }^{22}$ and the number of $\mathrm{k}$ points we used was 175 (fcc and bcc), $75\left(\alpha^{\prime \prime}\right), 52$ $(\alpha-\mathrm{U}), 72(\alpha-\mathrm{Np})$, and $16(\alpha-\mathrm{Pu})$. Hence, the calculation for Am in the $\alpha$-Pu structure was identical to the calculation we performed for plutonium recently, ${ }^{23}$ with exception of the atomic number (95 instead of 94). Total energy calculations were carried out for each crystal structure as a function of volume. These energies were then fitted to a Murnaghan equation of state which enabled us to calculate the Gibbs free energy,

$$
G=E+P V-T S=H-T S
$$

for the considered structures of Am. Here H, S, and E are the enthalpy, entropy, and internal energy of the system. In our calculations $\mathrm{T}=0$ and $\mathrm{E}$ is the total (electronic) energy. A phase transition between the two phases occurs if their Gibbs free energy coincides for a given pressure. Using the equation of state (pressure as a function of volume) for the two phases we are able to calculate the volume collapse associated with the transition.

\section{RESULTS}

Our main results are shown in Fig. 1. Fcc Am is calculated allowing for both spin and orbital polarization (GGA+OP) whereas the calculation of the other structures assumes spin degeneracy. The transition to the monoclinic $(\alpha-\mathrm{Pu})$ structure is calculated to occur at about $80 \mathrm{kbar}(8 \mathrm{GPa})$ and the volumes are $21.8 \AA^{3}$ and $16.3 \AA^{3}$ for the fcc and the monoclinic phase, respectively. The transition pressure is somewhat lower than the values (150-175 kbar) previously suggested by experimental work. ${ }^{10,11}$ The calculated $25 \%$ volume collapse is somewhat lower than what has been calculated previously ${ }^{15}(34 \%)$, where the $\alpha$-U structure was assumed to be high pressure structure, but considerably larger than the experimentally observed volume collapse between Am II and Am III ${ }^{11}$, but in better agreement with a transition from Am II to Am IV, see Fig. 2. In Fig. 2 we show the corresponding equation of state for our total energy calculations as obtained from our Murnaghan fits. Together with these results we also plot some experimental data reported by Heathman. ${ }^{11}$ The theoretical curves are fcc and monoclinic $(\alpha-\mathrm{Pu})$, respectively, whereas the experimental data represents Am I (dhcp), Am II (fcc), Am III ( $\alpha$ ") and $\operatorname{Am}$ IV $\left(\alpha^{\prime}\right)$. The pressure is plotted as a function of $V / V_{0}$ for all data. Theoretical $V_{0}$ depends upon which phase is considered, but here we decided to chose $V_{0}=26.85 \AA^{3}$, which is rather close to our (fcc in core) calculation, see below. This is also the theoretical equilibrium volume obtained from spin polarized GGA calculations (not shown) of dhcp Am and therefore seems to be an appropriate choice. The quantitative behavior is very similar between theoretical and experimental data. Close to the theoretical transition pressure, $80 \mathrm{kbar}$, the fcc calculations agree very well with experimental Am II (fcc) data as do our $\alpha$-Pu calculation with experimental Am IV $\left(\alpha^{\prime}\right)$ data. At lower pressure (close to zero) there is a discrepancy between theory and experiment that is rather serious. The equilibrium volumes, bulk moduli, and the pressure derivative of the bulk modulus are give in Table I. The fcc (GGA+OP) calculation gives an equilibrium too low compared to experimental dhcp equilibrium volume. For comparisons, we corrected for this discrepancy in the equilibrium volume by shifting the total energy curve so that $B$ and $B^{\prime}$ was 
unchanged but the equilibrium volume was identical to the experimental value $29.3 \AA^{3}$ (not shown). In this case, the Mott transition occurs instead already at about $44 \mathrm{kbar}$ and the volume collapse increases to about $40 \%$. Hence, correction for this discrepancy does not improve our theoretical agreement with experiment. It is unclear how to compare our theoretical transition pressure $(80 \mathrm{kbar})$ with experimental data because there is a large hysteresis in the experiments, the data by Heathman suggested that pure Am IV could be found at $175 \mathrm{kbar}$ upon compression, but during release there was an equal mix of Am IV and Am III at 59 kbar. It may be interpreted that our transition occur too early at $80 \mathrm{kbar}$. This might be due to underestimated total energy gain associated with the localization of the $5 f$ electrons in our calculations. Therefore, in another comparison, we artificially lowered the total energy curve for the (GGA+OP) calculation with $14 \mathrm{mRy}$ $(0.2 \mathrm{eV})$ and this resulted in a transition pressure close to $150 \mathrm{kbar}(15 \mathrm{GPa})$ with still a considerable $20 \%$ volume collapse. The calculated (GGA) zero-temperature equilibrium volumes of $\mathrm{Ce}$ and light actinides $(\mathrm{Th}-\mathrm{Pu})^{24,25}$ are on average about $7 \%$ smaller than measured room temperature data, with the worst case being Th with a $10 \%$ discrepancy. It therefore seems likely that also the itinerant monoclinic phase $(\alpha-\mathrm{Pu})$ of Am has a too low calculated equilibrium volume in the present calculations. The equilibrium volume is calculated to be $16.9 \AA^{3}$. If we introduce a correction so that the monoclinic phase obtains a $7 \%$ larger equilibrium volume, we instead obtain a transition pressure of about $100 \mathrm{kbar}(10 \mathrm{GPa})$ and a volume collapse of about $18 \%$. This correction gives a somewhat better agreement with experiment for the transition pressure whereas the calculated volume collapse is rather insensitive to this correction.

Experimentally ${ }^{10,11}$ there were proposed an orthorhombic $(\alpha-\mathrm{U})$ at about $150 \mathrm{kbar}(15$ $\mathrm{GPa}$ ) in Am. In our calculation this orthorhombic structure (with $b / a, c / a$, and atomic coordinate $y$ set equal to their equilibrium values for uranium) is substantially higher in energy than the monoclinic phase and provided our description of the electronic structure is accurate, we therefore rule out the orthorhombic phase in the high pressure/low temperature phase diagram of Am. If, hypothetically, a transition to the $\alpha$-U phase would occur, completely neglecting the monoclinic $(\alpha-\mathrm{Pu})$ phase, the transition pressure is calculated to occur at about $200 \mathrm{kbar}(20 \mathrm{GPa})$ accompanied by a volume collapse of about $21 \%$.

In Table I we summarize our equation of state data for the calculated crystal structures. The Murnaghan fit of fcc Am gave a bulk modulus ( $B$ ) of about 430 kbar (43 GPa) and a $B^{\prime}$ equal to 2.9. The equilibrium volume is too low, only $25.1 \AA^{3}$ compared to the observed volume of $29.3 \AA^{3}$, but the bulk modulus is in rather good agreement with experiment. Our calculations underestimate the equilibrium volume with about $14 \%$ which may indicate that the $5 f$ contribution to the chemical bond is overestimated in our GGA+OP scheme at lower pressures. The large discrepancy for the equilibrium volume is a serious failure of the theory, but is consistent with the results found for $\operatorname{Pr}$ recently, ${ }^{18}$ where the difference between theory and experiment for the equilibrium volume was about $14 \%$. It is possible to remove the $5 f$ bonding by putting these electrons in ad hoc to the core. In Fig. 3 we compare calculations for fcc Am (GGA+OP) with non polarized (GGA) calculations with the $5 f$ states treated as core states ( $5 f$ in core). The ( $5 f$ in core) calculation is shifted down an amount $0.17 \mathrm{Ry}(2.3 \mathrm{eV})$ to enable a more clear comparison between the two energy curves. The equilibrium volume for the ( $5 f$ in core) calculation is in somewhat 
better agreement, $26.6 \AA^{3}$, but still almost $10 \%$ too low compared to experiment. The corresponding bulk modulus is about $460 \mathrm{kbar}(46 \mathrm{GPa}$ ) in rather close agreement with our (GGA+OP) calculation. Also $B^{\prime}$ is in good agreement with the (GGA+OP) theory, 3.4 compared to 3.0. From Fig. 3 we conclude that for the volume range close to the equilibrium the two theoretical treatments $(\mathrm{GGA}+\mathrm{OP})$ and ( $5 f$ in core) are in relatively good agreement, with a small discrepancy of about $4 \%$ in their respective equilibrium volume. Notice, however, that for compressed volumes the total energy curves begin to separate between the two calculations. This is certainly expected because the ( $5 f$ in core) treatment should become less satisfactory at higher pressures. We anticipate an increased overlap between the $5 f$ orbitals at smaller volumes which eventually will form band states. At this point, it would of course be grossly inaccurate to treat them as core states. This effect is inherent in the $(\mathrm{GGA}+\mathrm{OP})$ theory where a suppression of the magnetic moments signal a delocalization.

In Fig. 4 we show the spin, orbital, and total magnetic moments as a function of atomic volume for fcc Am calculated using the (GGA+OP) approach. The orbital moment is enhanced by the orbital polarization of the $5 f$ orbitals and at the equilibrium volume it is about -0.85 Bohr magnetons. The majority contribution to the orbital moment is traced to the $5 f$ spin down states $(-0.93)$ with a small contribution also from the $6 d$ spin down $(0.13)$ and spin up $(-0.05)$ states. With the orbital polarization switched off the orbital moment is smaller in magnitude ( -0.65 Bohr magnetons). The $5 f$ band is less than half full and therefore the sign of the spin-orbit coupling (corresponding to Hund's third rule of an open shell atom) turns the orbital moment antiparallel to the spin moment. The total and spin magnetic moments are slowly decreasing in magnitude with volume whereas the orbital moment is almost constant until about $17 \AA^{3}$ where both spin and orbital moment collapse to zero. This signals a complete $5 f$ delocalization in Am and $5 f$ band states that contribute to the chemical bonding between atoms. At this volume the $5 f$ states in Am are itinerant, similar to the lighter actinides, Th-Pu. Consequently, in Fig. 1, fcc $\mathrm{Am}$ is the most unfavorable structure at this volume and instead the monoclinic $(\alpha-\mathrm{P} u)$ structure has the lowest energy. This result confirms the simple model calculations carried out by Söderlind et al. ${ }^{12}$ who showed that for a $5 f$ band occupation of about six, the $\alpha$-Pu structure should be lower than both the $\alpha$-U and fcc structures.

The orbital polarization energy, the $\frac{1}{2} E_{\sigma}^{3} L_{\sigma}^{2}$ term, was of the order of 2-7 mRy throughout the studied volume range. The Racha parameter, $E_{\sigma}^{3}$, is a linear combination of Slater integrals and was in our calculations for Am of the order of 4-6 mRy. Calculations without orbital polarization gave a somewhat lower transition pressure (65 kbar) and a somewhat larger volume collapse (28\%).

\section{DISCUSSION}

We have studied five crystal structures of Am with a first-principles method using the (GGA+OP) scheme. The total energy for four of these structures (bcc, $\alpha$-U, $\alpha$-Np, and $\alpha$-Pu) was calculated assuming spin degeneracy whereas for the fcc structure, this requirement was lifted. At $80 \mathrm{kbar}$ we calculate a transition from fcc Am to monoclinic Am and a volume collapse of $25 \%$. We interpret this transition as a Mott transition, the onset of a low symmetry crystal structure is prompted by a delocalization of $5 f$ electrons 
in Am. The low density fcc phase is also modeled by a calculation with the $5 f$ electrons occupying core states. For low pressures this rather ad hoc approximation is in relatively good agreement with the (GGA+OP) calculations with a very similar $B$ and $B^{\prime}$ but a $4 \%$ larger equilibrium volume. With increasing pressure the treatment with $5 f$ electrons in the core becomes gradually inappropriate with an inaccurate total energy as a result.

Calculations of the transition pressure between fcc and monoclinic Am are sensitive to the accuracy of the total energy for both the localized and the itinerant phase. The transition pressure would be considerably higher and the volume collapse smaller if the equilibrium volume for the monoclinic phase was $5-10 \%$ larger. This is certainly within the usual error associated with a GGA calculation for an $f$ electron metal. The transition pressure would also increase considerably upon a small downward shift (0.1-0.2 eV) of the energy curve for the low density fec phase. Thus, inaccuracies in the calculations could easily explain the fact that we calculate a transition pressure somewhat lower than the values reported for this transition. A large volume collapse, however, seems relatively insensitive to possible inaccuracies in the total energy calculations and we therefore have confidence in this result. We appreciate the difficulties involved in determine the correct crystal structure from high pressure experiments and the necessary fitting that has to be done. Also the hysteresis in the experimental results make it hard to directly compare our results with experiment. We believe, however, that Fig. 2 is a clear evidence that our technique is able to describe the correct physics of the high pressure transitions in Am. To get a more accurate description over all the exchange/correlation functional needs to be improved.

In the present paper we have investigated the total energy of two different configurations; delocalized $5 f$ states and localized, chemically inert $5 f$ states. Provided there are no complications involving other electronic configurations such as mixed valence, Kondo behavior and so on, we rule out the $\alpha$-U structure as the high pressure phase of Am. Calculations of the type presented here seldom give the wrong structural stability and in our case we find that $\alpha$-U structure is $\sim 20 \mathrm{mRy}$ higher in energy than the lowest energy structure, $\alpha$-Pu. This is a rather large energy difference. Also the $\alpha^{\prime \prime}$ structure, although not relax with respect to its internal parameters, show very high energies compared to the $\alpha$-Pu structure. As regards the stability of the $\alpha$-Pu structure, we mention that there are other structure-types, we have not investigated which may be lower in energy. However, a blind search for these structures, without experimental input is beyond current computational capabilities.

\section{ACKNOWLEDGMENTS}

A. K. McMahan is acknowledged for many discussions regarding the $f$ localization problem. Work performed under the auspices of the U.S. Department of Energy by the Lawrence Livermore National Laboratory, contract number W-7405-ENG-48. R. A., O. E. and B. J. wish to thank the Swedish Natural Science Research Council (NFR) for financial support and the Swedish Material Consortium No.9 financed by NUTEK and NFR. 


\section{REFERENCES}

${ }^{1}$ B. Johansson and M. S. S. Brooks in Handbook on the Physics and Chemistry of the Actinides, edited by K.A. Gschneidner, Jr., L. Eyring, G. H. Lander and G. R. Choppin (North-Holland, Amsterdam, 1993)

${ }^{2}$ K. A. Gschneidner, Jr., Solid State Physics (Academic, New York, 1964) Vol. 16.

${ }^{3}$ O. Eriksson, P. Söderlind, and J. M. Wills, Phys. Rev. B 45, 12588 (1992).

${ }^{4}$ J. C. Duthie and D. G. Pettifor, Phys. Rev. Lett. 38, 564 (1977); A. K. McMahan, H. L. Skriver, and B. Johansson, Phys. Rev. B 23, 5016 (1981).

${ }^{5}$ B. Johansson, Phys. Rev. B 11, 2740 (1975).

${ }^{6}$ O. Eriksson and J. M. Wills (unpublished)

${ }^{7}$ J. Akella, Q. Johnson, W. Thayer, R. N. Schock, J. of less. comm. metals 68, 95 (1979); J. Akella, Q. Johnson, R. N. Schock, J. of Geophys. Res. 85, 7056 (1980).

${ }^{8}$ L. B. Asprey, U. B. Benedict, J. R. Peterson, J. C. Spirlet, Rep. Workshop on Actinides under pressure, Karlsruhe, p. 9 (1983).

${ }^{9}$ U. B. Benedict, J. P. Itie, C. Dufour, S. Dabos, J. C. Spirlet, Americium and Curium chemistry and technology (D. Riedel publishing company, 1985) p. 213.

${ }^{10}$ U. Benedict, J. Phys. Colloq. (Paris), C8-145 (1984).

${ }^{11} \mathrm{~S}$. Heathman (unpublished)

${ }^{12}$ P. Söderlind, J. M. Wills, and O. Eriksson, Phys. Rev. B 57, 1320 (1998).

${ }^{13}$ D. Pettifor, Bonding and Structure of Molecules and Solids (Oxford Science Publications, Clarendon Press, Oxford 1995).

${ }^{14}$ H. L. Skriver, B. Johansson, and O. K. Andersen Phys. Rev. Lett. 41, 42 (1978).

${ }^{15}$ O. Eriksson and J. M. Wills, Phys. Rev. B 45, 3198 (1992).

${ }^{16}$ M. S. S. Brooks, Physica (Amsterdam) 130B, 6 (1985).

${ }^{17}$ O. Eriksson, B. Johansson, and M. S. S. Brooks, J. Phys. Condens. Matter 1, 4005 (1989).

18 A. Svane, J. Trygg, B. Johansson, and O. Eriksson, Phys. Rev. B 56, 7143 (1997).

19 J. Donohue, The Structure of the Elements, (Wiley, New York, 1974.)

${ }^{20}$ J.S. Olsen, L. Gerward, U. Benedict, and J.-P. Itie', Physica 133B, 129 (1985).

${ }^{21}$ J. M. Wills (unpublished); J. M. Wills and B. R. Cooper, Phys. Rev. B 36, 3809 (1987);

D. L. Price and B. R. Cooper, Phys. Rev. B 39, 4945 (1989).

${ }^{22}$ D.J. Chadi and M.L. Cohen, Phys. Rev. B 8, 5747 (1973); S. Froyen, ibid, 3168 (1989).

${ }^{23}$ P. Söderlind J.M. Wills, B. Johansson, and O. Eriksson, Phys. Rev. B 55, 1997 (1997).

${ }^{24}$ P. Söderlind, O. Eriksson, J. M. Wills, and B. Johansson, Phys. Rev. B 50, 7291 (1994).

${ }^{25}$ P. Söderlind, Adv. in Phys. 47, 959 (1998). 


\section{FIGURES}

FIG. 1. Total energies (+61046 Ry/atom) for Am in the bcc, fcc, $\alpha^{\prime \prime}, \alpha-\mathrm{U}, \alpha-\mathrm{Np}$, and $\alpha$-Pu structures. The fcc phase is calculated using spin and orbital polarization. The other structures are calculated assuming spin degeneracy. The ratios between the crystal structure parameters for the $\alpha^{\prime \prime}, \alpha$-U, $\alpha$-Np, and $\alpha$-Pu structures are kept equal to their equilibrium values for $\mathrm{Ce}$ (proposed), U, Np, and $\mathrm{Pu}$.

FIG. 2. Equation of state as obtained from Murnaghan fits to the total energies for fcc and monoclinic $(\alpha-\mathrm{Pu})$ structures. Results indicate a volume collapse of $25 \%$ at $80 \mathrm{kbar}(8 \mathrm{GPa}$ ) pressure from the fcc to the monoclinic phase. Also experimental data from Heathman ${ }^{11}$.

FIG. 3. Two different treatments of the localized fec phase of Am. The (GGA+OP) treatment, used in the present calculations, compared to a calculation where the $5 f$ electrons are treated as core electrons. The latter calculation is shifted down an amount of $0.17 \mathrm{Ry}(2.3 \mathrm{eV})$ to enable a more visual comparison.

FIG. 4. Spin, orbital, and total magnetic moment (Bohr magnetons) as obtained from the $(\mathrm{GGA}+\mathrm{OP})$ calculation of fec Am. 


\section{TABLES}

TABLE I. A compilation of equation of state data for americium. The equilibrium volume is given in $\AA^{3}$ and the bulk modulus $B$ in kbar. Fcc results are obtained from spin polarized calculations including orbital polarization $(\mathrm{GGA}+\mathrm{OP})$ and with the $5 f$ electrons treated as core electrons ( $5 f$ in core).

\begin{tabular}{cccc}
\hline \hline Crystal structure & $V_{0}$ & $B$ & $B^{\prime}$ \\
\hline fcc (GGA+OP) & 25.1 & 430 & 2.9 \\
fcc (5f in core) & 26.6 & 460 & 3.4 \\
$\alpha-\mathrm{Pu}$ & 16.9 & 1790 & 8.0 \\
$\alpha-\mathrm{Np}$ & 17.0 & 1990 & 5.8 \\
$\alpha-\mathrm{U}$ & 17.1 & 1540 & 5.7 \\
$\alpha^{\prime \prime}$ & 17.8 & 1210 & 6.4 \\
bcc & 16.5 & 1560 & 6.5 \\
dhcp (expt) & 29.3 & $400-450$ & 6.0 \\
fcc (expt) & 29.3 & 294 & 3.0 \\
\hline \hline
\end{tabular}




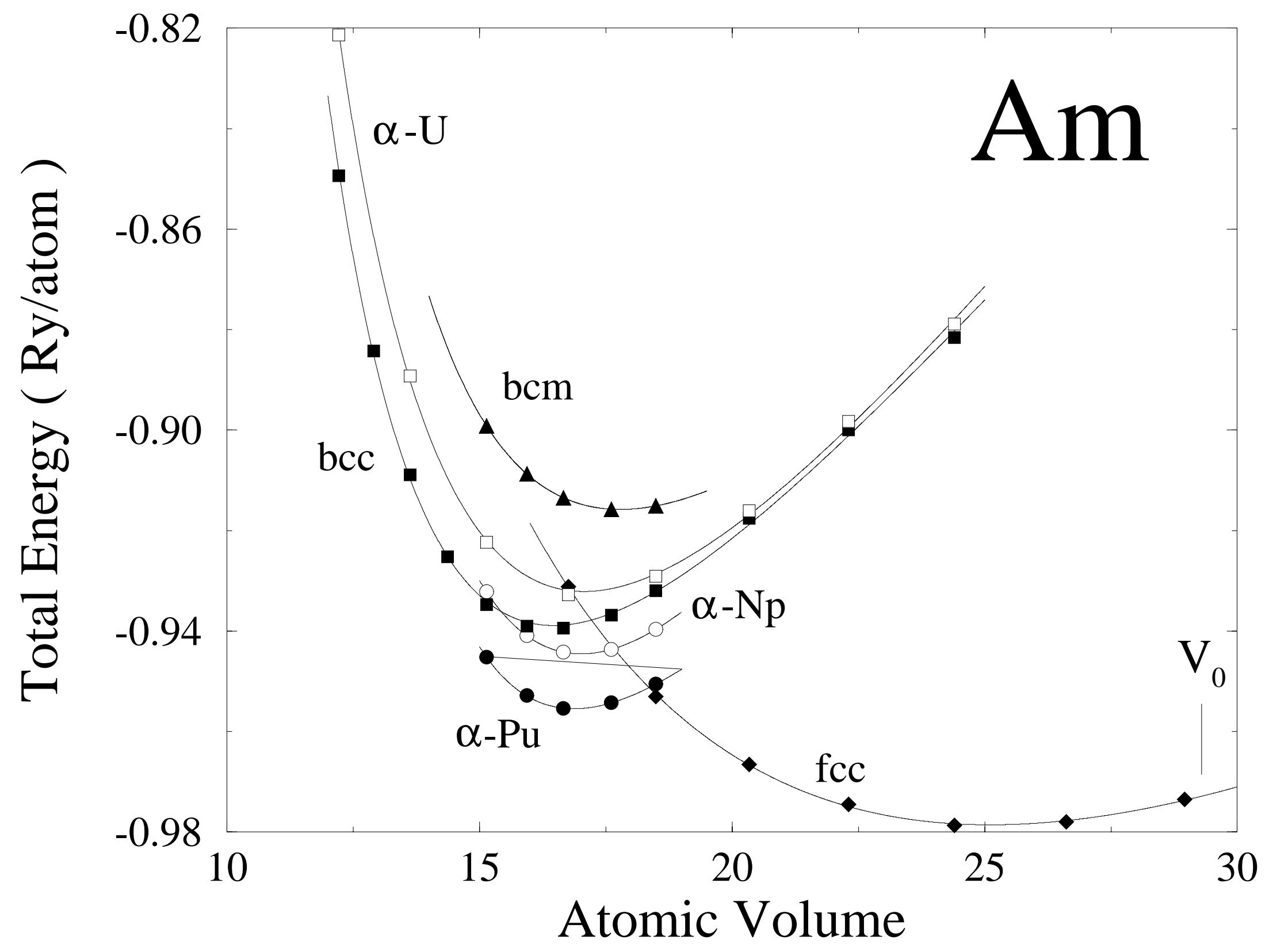




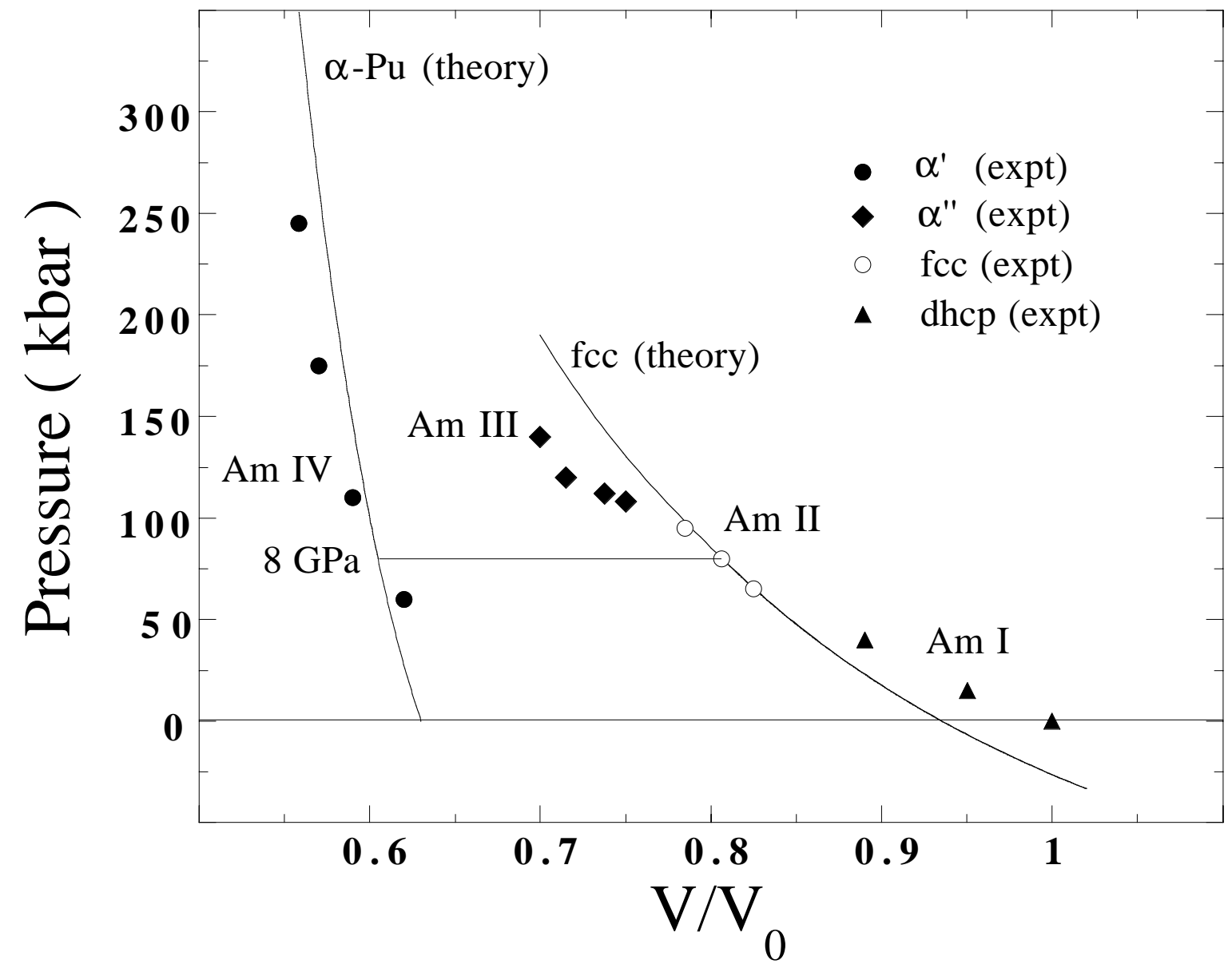




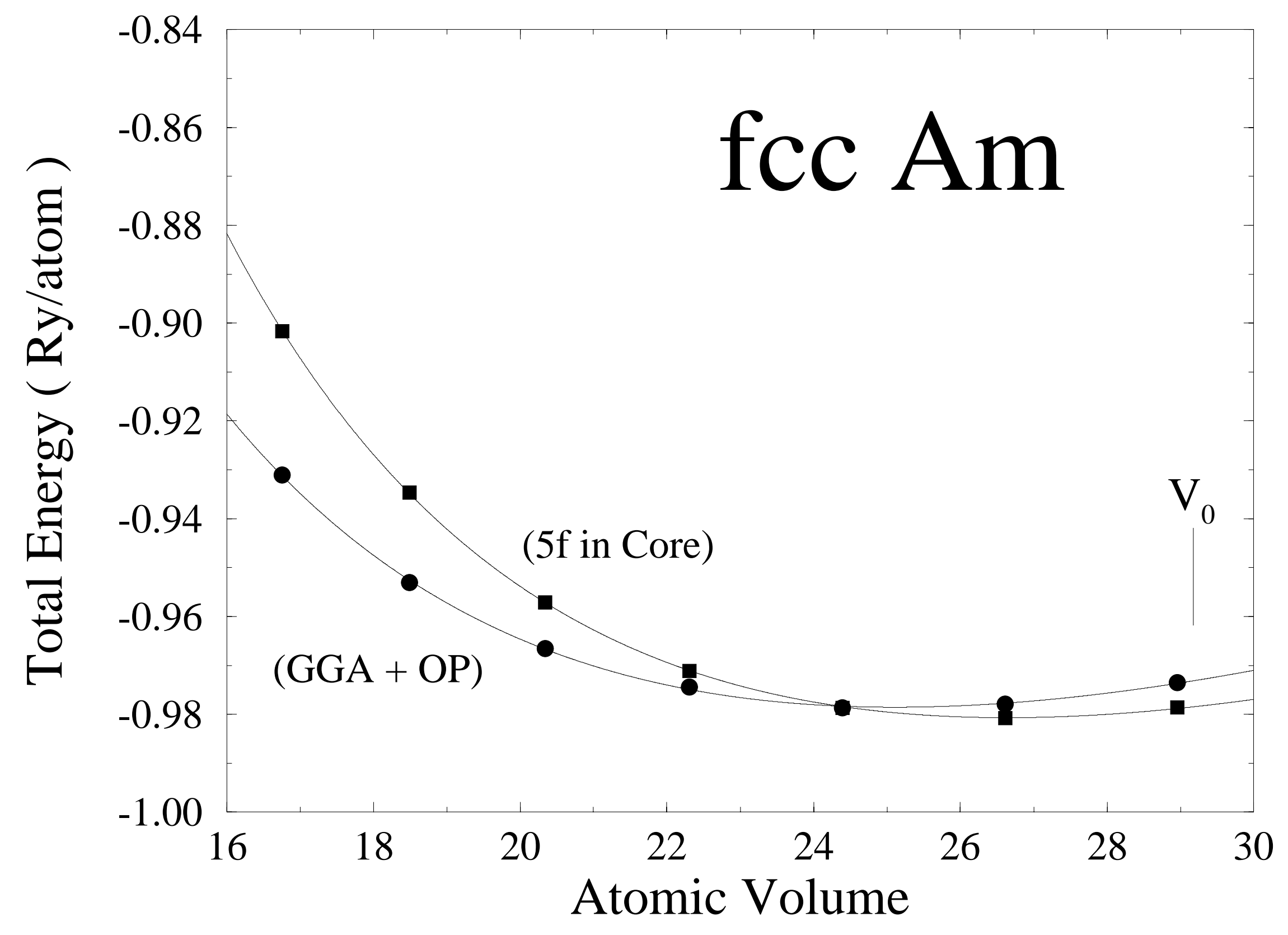




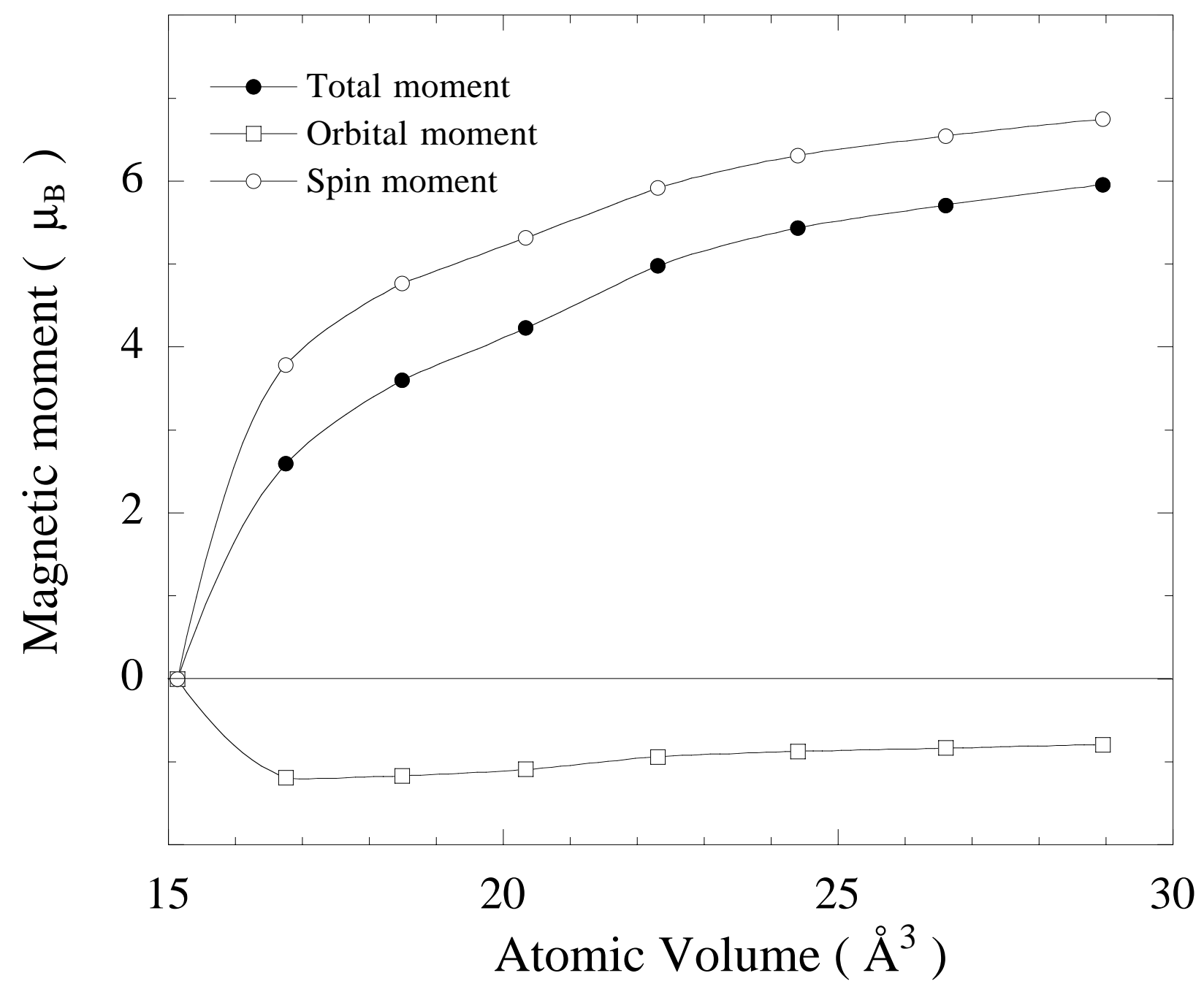

